\title{
Interleukin-8 Levels in the Stratum Corneum as a Biomarker for Monitoring Therapeutic Effect in Atopic Dermatitis Patients
}

\author{
Susumu Murata Sakae Kaneko Eishin Morita \\ Department of Dermatology, Shimane University Faculty of Medicine, Izumo, Japan
}

\section{Keywords}

Atopic dermatitis $\cdot$ Biomarker $\cdot$ Interleukin-8 $\cdot$ Stratum corneum $\cdot$ Tape-stripping method

\begin{abstract}
Introduction: The stratum corneum contains several growth factors and cytokines that are synthesized in keratinocytes. We previously reported that the amount of interleukin-8 in the stratum corneum (sclL-8) is related to the severity of local skin inflammation in atopic dermatitis (AD). However, it is unknown whether sclL-8 levels reflect pharmacologic responses to a therapeutic intervention in AD patients. Therefore, in this study, we aimed to investigate whether the improvement of dermatitis in AD is correlated with sclL-8 levels before and after topical corticosteroid treatment. Methods: Stratum corneum samples were collected from 22 AD patients using the noninvasive tape-stripping method before treatment, 2 weeks after topical treatment, and 4-6 weeks after treatment. Results: sclL-8 levels on the forearm reduced significantly from $790 \pm 348 \mathrm{pg} / \mathrm{mg}$ before treatment to $163 \pm 68 \mathrm{pg} / \mathrm{mg} 2$ weeks after treatment and $100 \pm 37 \mathrm{pg} /$ mg 4-6 weeks after corticosteroid treatment. sclL-8 levels on the abdomen also reduced significantly from $902 \pm 391$ to $142 \pm 38 \mathrm{pg} / \mathrm{mg}$ at the end of study. The reduction in sclL-8 levels was associated with the improvement in local skin se-
\end{abstract}

karger@karger.com www.karger.com/iaa

Karger $\stackrel{\text { ' }}{5}$

GOPEN ACCESS
(C) 2021 The Author(s)

Published by S. Karger AG, Basel

This is an Open Access article licensed under the Creative Commons Attribution-NonCommercial-4.0 International License (CC BY-NC) (http://www.karger.com/Services/OpenAccessLicense), applicable to the online version of the article only. Usage and distribution for commercial purposes requires written permission. verity in AD. We also found that sclL-8 levels, along with blood biomarker levels (serum thymus and activation-regulated chemokine, lactate dehydrogenase, and \%eosinophil), decreased significantly after the treatment. Conclusion: The sclL-8 concentration decreases with improvements in skin symptoms in AD patients after topical corticosteroid treatment; thus, it may be a suitable biomarker for monitoring therapeutic effects in $A D$ patients.

(c) 2021 The Author(s).

Published by S. Karger AG, Basel

\section{Introduction}

Atopic dermatitis (AD) is a relapsing chronic inflammatory skin disorder that affects children and adults and is considered one of the most common chronic skin diseases, with an estimated global prevalence of 230 million $[1,2]$. Several serum biomarkers have been used to evaluate the severity of AD. Of these, serum thymus and activation-regulated chemokine (TARC) is currently one of the most reliable biomarkers [3-5]. Serum lactate dehydrogenase $(\mathrm{LDH})$ and eosinophil count are other biomarkers that correlate with AD severity [6]. Despite the

Edited by: H.-U. Simon, Bern.
Susumu Murata

Department of Dermatology, Shimane University Faculty of Medicine Izumo, Enya-cho 89-1

Shimane 693-8501 (Japan)

smurata@med.shimane-u.ac.jp 
vital information that these serum biomarkers provide in the evaluation of $\mathrm{AD}$, their measurement requires blood sampling; therefore, frequent measurements are not feasible. Recently, the tape-stripping technique was developed for noninvasive determination of the concentrations of cytokines and chemokines in the stratum corneum of cutaneous lesions [7]. Such measurements should reflect the inflammatory condition of the affected skin. Many cytokines and chemokines have been investigated for use as biomarkers of the severity of AD.

We previously reported that the amount of TARC in the stratum corneum (scTARC) is correlated with the severity of cutaneous lesions, especially the acute inflammatory signs, such as erythema, edema, papules, and oozing or crusts $[8,9]$. scTARC is also correlated with the systemic severity of $\mathrm{AD}$, as evaluated using the Severity Scoring of Atopic Dermatitis (SCORAD) index, serum TARC levels, serum total immunoglobulin E (IgE) levels, and blood eosinophil counts. However, scTARC is evaluated semi-quantitatively using an immunofluorescent technique as scTARC content is too low for quantification using an ELISA. As the immunofluorescent method is time and labor intensive, it is impractical for routine monitoring purposes.

Subsequently, we have used commercially available ELISAs to evaluate various cytokines and growth factors in the stratum corneum $[10,11]$. We used the tape-stripping method for the noninvasive collection of stratum corneum samples and evaluated cytokines and growth factors that are considered to play a role in the inflammation of the skin. This included several interleukins (ILs); tumor necrosis factor- $\alpha$; chemokine ligand 5 (RANTES); eotaxin; monocyte chemoattractant protein-1; macrophage inflammatory proteins- $1 \alpha$ and $1 \beta$; granulocyte, macrophage, and granulocyte-macrophage colony-stimulating factor; nerve growth factor; vascular endothelial growth factor; and transforming growth factor (TGF)- $\alpha$ and TGF- $\beta$.

As a result, we discovered that IL-8, IL-18, vascular endothelial growth factor, and TGF- $\alpha$ were present in sufficient amounts to be measured using commercially available ELISAs and further evaluated their association with cutaneous symptoms $[10,11]$. Of these cytokines, the amount of IL- 8 in the stratum corneum (scIL-8) demonstrated the highest correlation coefficient with the cutaneous symptoms. Based on these observations, we speculated that scIL-8 level is a significant biomarker in evaluating cutaneous conditions as well as general disease severity in AD. However, whether scIL-8 concentration will reflect pharmacologic responses to $\mathrm{AD}$ symptom

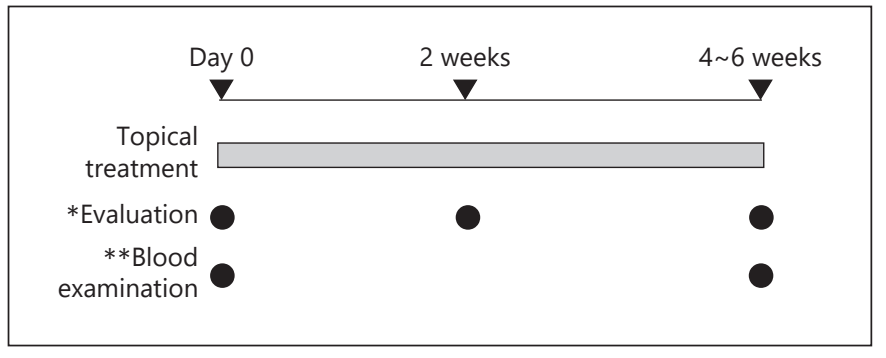

Fig. 1. Study design. *Evaluation included skin score, TEWL, and skin water content. ${ }^{* *}$ Blood examination includes white blood cell count, \%eosinophil, serum LDH, serum TARC, and serum total IgE. TEWL, transepidermal water loss; LDH, lactate dehydrogenase; TARC, thymus and activation-regulated chemokine.

treatment remains unclear. Although several therapeutic options are available for the treatment of $\mathrm{AD}$, the preferred first-line therapy is topical corticosteroid [12-14]. The aim of this study was to evaluate the changes in scIL8 before and after topical corticosteroid treatment in patients with $\mathrm{AD}$, to evaluate the correlation between change in scIL-8 level and improvements in skin symptoms, and to determine whether scIL- 8 can be used as a biomarker to monitor disease activity in $\mathrm{AD}$.

\section{Methods}

Study Design and Patients

We enrolled 22 patients (11 males and 11 females) from Shimane University Hospital who met the diagnostic criteria for AD established by the Japanese Dermatological Association [14]. Topical corticosteroid treatment was administered for 4-6 weeks (Fig. 1). Evaluation was performed at day 0 (first visit), 2 weeks later (second visit), and 4-6 weeks later (third visit), and blood examination was performed at the first and third visits. Patients undergoing systemic immunosuppressive therapy were excluded. This study was approved by the Ethics Committee of Shimane University Faculty of Medicine (Approval No. 1473) and was performed in accordance with the Declaration of Helsinki. The study design was fully explained to the patients, and written informed consent was obtained from them.

\section{Topical Treatment}

The AD patients were instructed to use daily topical corticosteroid ointments containing betamethasone butyrate propionate (Antebate ${ }^{\circledR}$; Torii Pharmaceutical Co., Ltd, Tokyo, Japan). One fingertip unit of topical corticosteroid was suggested for use in an area of the skin twice the size of the palm of the patient's hand. Depending on their symptoms, patients were allowed to use routine therapy including moisturizer ointment and antihistamines; however, no systemic treatment (oral corticosteroid or cyclosporine) was allowed during the test period. 


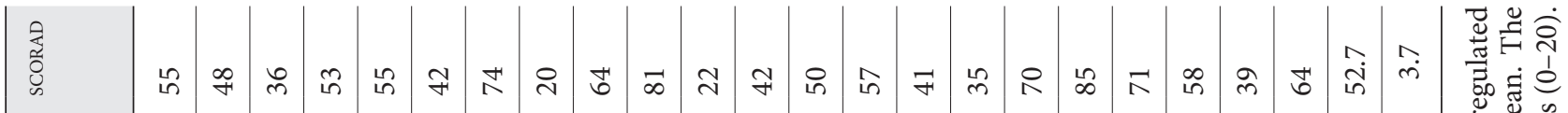

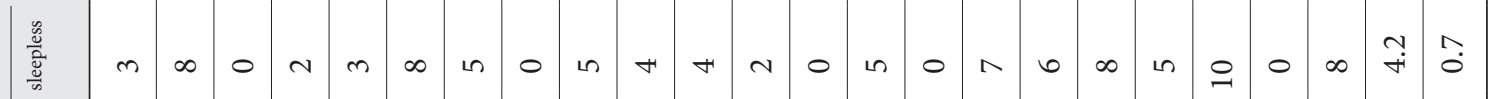
$u$ U

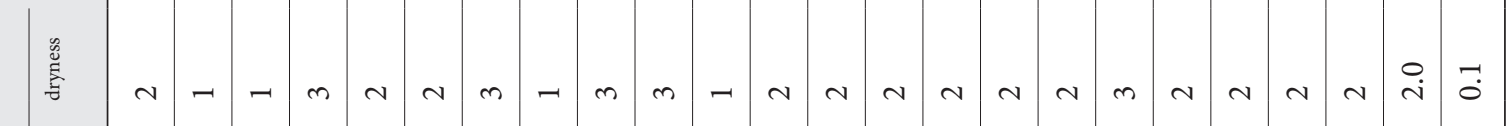
产

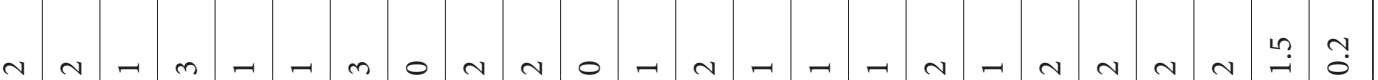

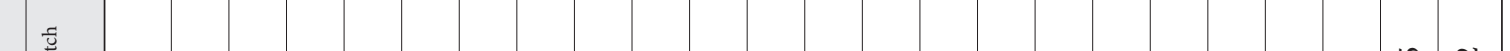

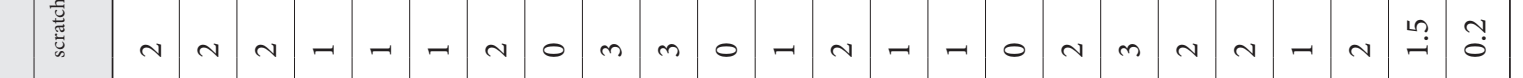

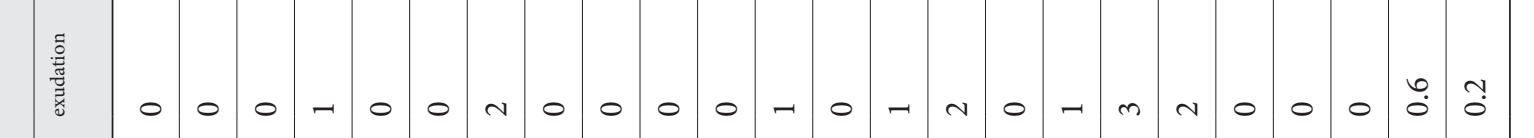

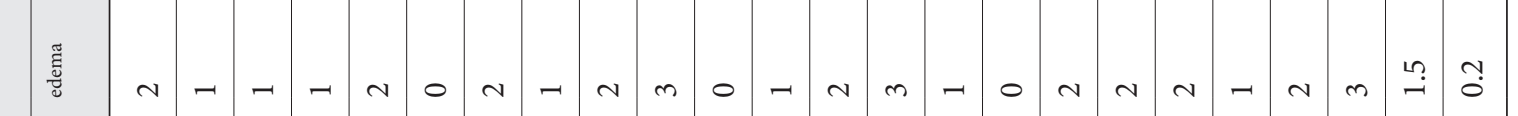

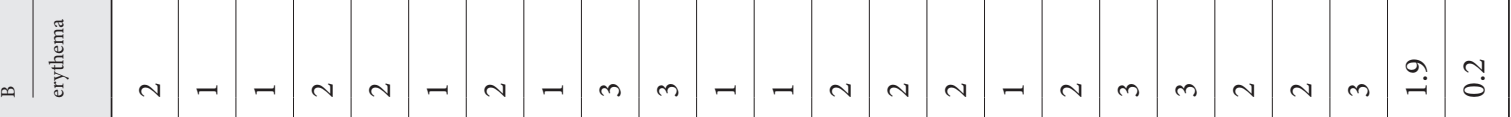

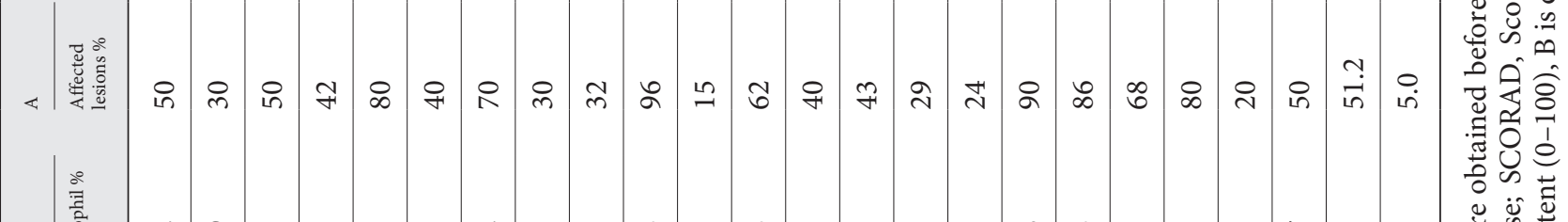

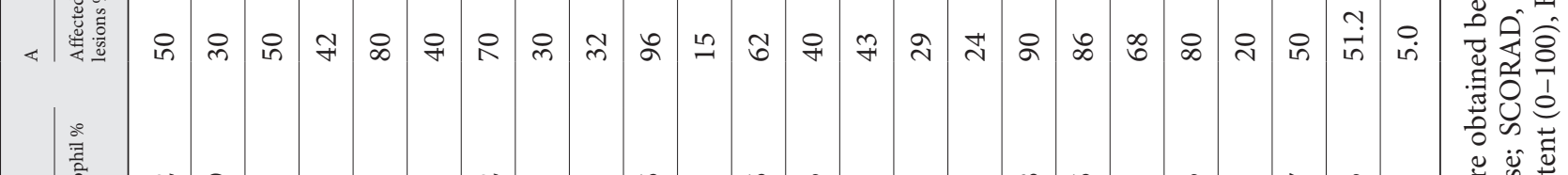

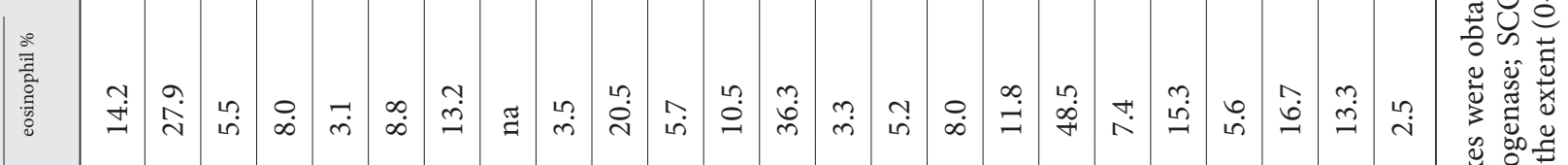

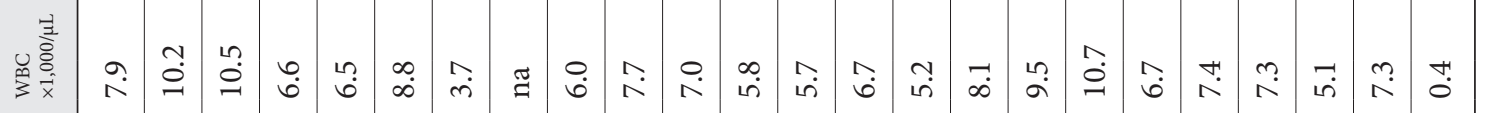

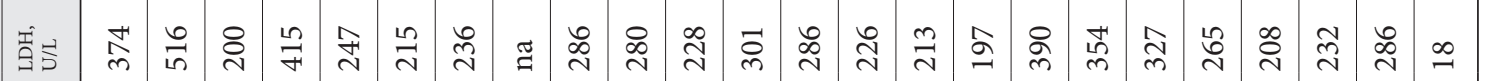

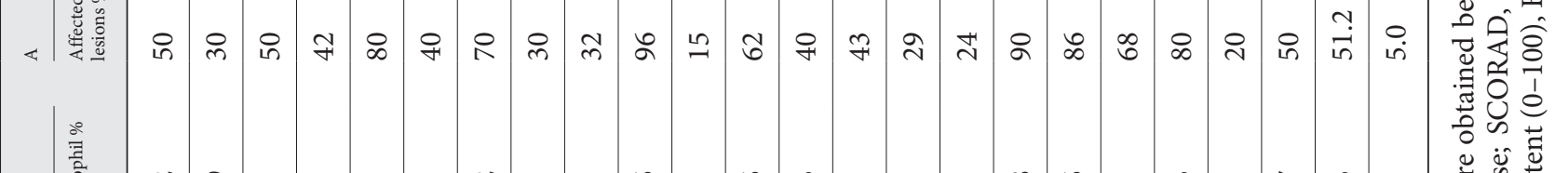

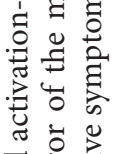
宅氙总 范 茪 空苛焉

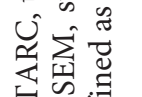
نे

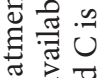
氖芯 듕 क्ष त्ञ 过泀 ज्ञّ̆ ㄴ.

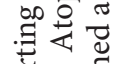
要品 氙. उ 월 谣 焉

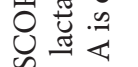
年 ت્ن

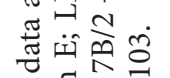




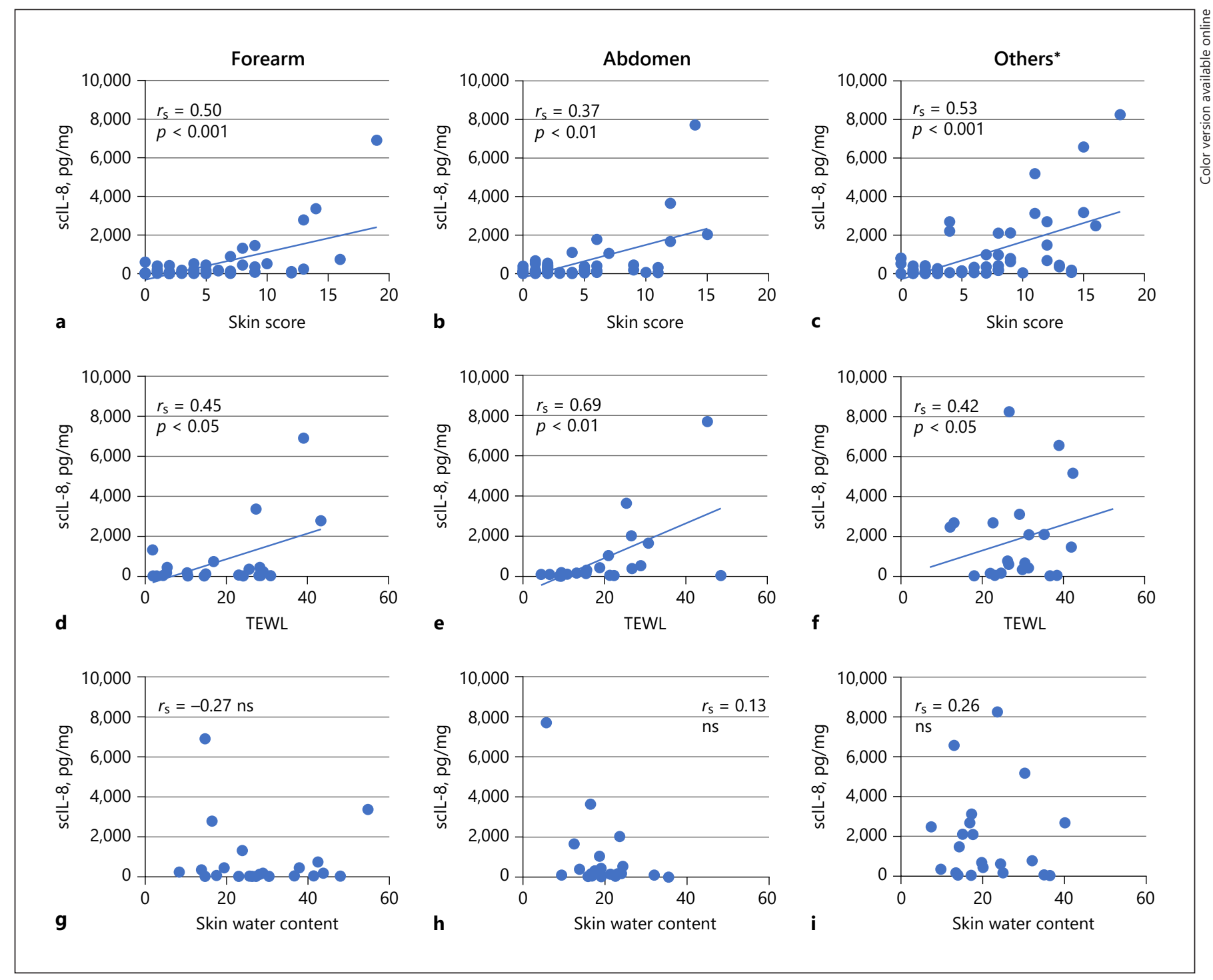

Fig. 2. Pre-treatment correlations in atopic dermatitis patients between scIL-8 concentration and skin score, TEWL, and skin water content of the forearm, abdomen, and area with the most severe symptoms (others*). There were significant correlations between scIL- 8 and skin score and between scIL- 8 and TEWL but not between scIL-8 and skin water content. scIL-8, stratum corneum interleukin-8; TEWL, transepidermal water loss.

\section{Evaluation of Cutaneous Lesion Conditions}

Three sites were chosen for the evaluations - the inside of the forearm, abdomen, and area with the most severe symptoms in each patient. Skin scores were assessed visually for each of the 3 skin sites to assess the severity of the disease using 7 SCORAD index parameters (erythema, edema, lichenification, oozing/exudation, excoriation, xerosis/dryness, and itch) [14]. According to increasing symptom severity, each parameter was scored from 0 to 3 , for a total possible score of 21 . Before tape stripping, transepidermal water loss (TEWL) and skin water content were measured at each skin site in an airconditioned room using the Corneometer ${ }^{\circledR}$ CM825 and
Tewameter ${ }^{\circledR}$ MPA5 (Courage + Khazaka electronic GmbH, Cologne, Germany), respectively.

\section{Blood Examination}

Blood was collected at the first and third visits to assess the white blood cell count, \%eosinophil, serum levels of $\mathrm{LDH}$, total IgE, and TARC.

Tape Stripping of the Stratum Corneum

Tape stripping was performed on the cutaneous sites using plastic tape $\left(24 \mathrm{~mm} \times 5 \mathrm{~cm}\right.$, Cellotape ${ }^{\circledR}$; Nichiban, Tokyo, Japan) $[10,11]$ after the sites were cleaned with ethanol. Plastic tape was 


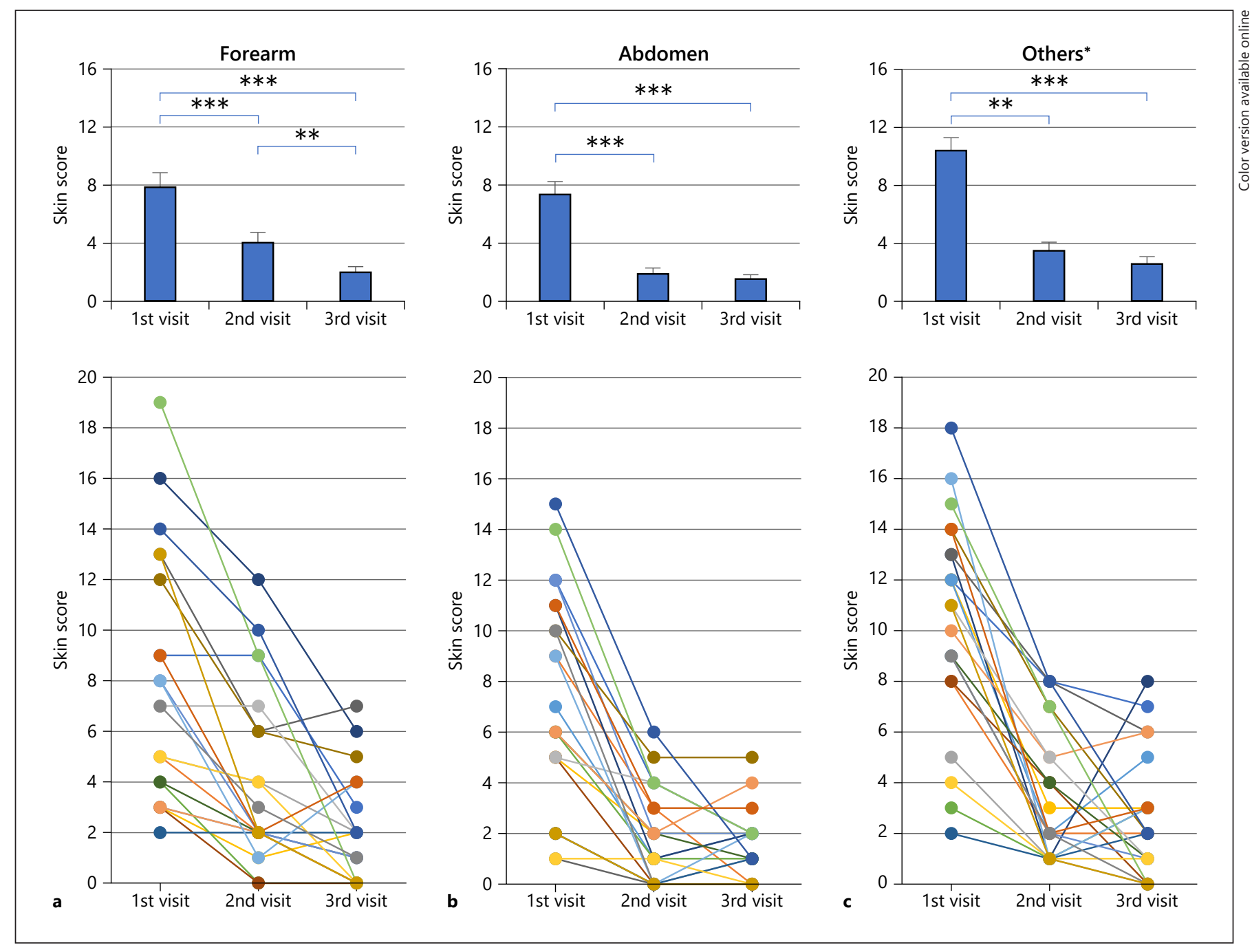

Fig. 3. Change in skin scores before, during, and after topical treatment. Skin scores were evaluated at the first, second, and third visits for the forearm, abdomen, and area with the most severe symptoms (others*). The average data are indicated by the bar graphs at the top, and individual data are indicated by the line graphs below. Data are expressed as the mean \pm SEM. ${ }^{* *} p<0.01,{ }^{* * *} p<0.001$. SEM, standard error of the mean.

applied to the skin, pressed for approximately $10 \mathrm{~s}$, and removed gently; the same procedure was repeated 5 times. The pieces of tape were stored at $-20^{\circ} \mathrm{C}$ until further analysis.

\section{Measurement of scIL-8}

scIL-8 was evaluated using the method described previously $[10,11]$. The tape-stripping samples were briefly immersed in $5 \mathrm{~mL}$ of hexane. After centrifugation $(3,000 \mathrm{rpm}, 15 \mathrm{~min}$ at $4^{\circ} \mathrm{C}$ ), the supernatant, containing tape glue and miscellaneous chemicals, was removed. The remaining samples were again subjected to centrifugation $\left(15,000 \mathrm{rpm}, 15 \mathrm{~min}\right.$ at $\left.4^{\circ} \mathrm{C}\right)$ followed by the addition of $1 \mathrm{~mL}$ of hexane. The precipitants, containing the corneal layers, were collected. Proteins were extracted in $1 \mathrm{~mL}$ of extraction buffer $(0.1 \mathrm{M}$ Tris- $\mathrm{HCl}, \mathrm{pH} 8.0$, and $0.5 \%$ Triton $\mathrm{X}-100$ ) under ultrasound sonification (Branson Sonifier ${ }^{\circledR} 450$; Emerson Japan, Ltd., Atsugi, Japan) for 3 min. The supernatants were purified using 4-mm filters (Millex ${ }^{\circledR}$; Millipore, Tokyo, Japan) and subjected to centrifugation $\left(15,000 \mathrm{rpm}, 15 \mathrm{~min}\right.$ at $\left.4^{\circ} \mathrm{C}\right)$. scIL- 8 in the purified supernatants was analyzed using ELISA kits (Human IL-8/CXCL8 Quantikine ${ }^{\circledR}$ ELISA; R\&D Systems, Minneapolis, MN, USA). The total protein contents were measured using the DC protein assay (Bio-Rad Laboratories, Inc., Hercules, CA, USA). scIL- 8 concentration was expressed as pg per $\mathrm{mg}$ of protein content of the stratum corneum. 

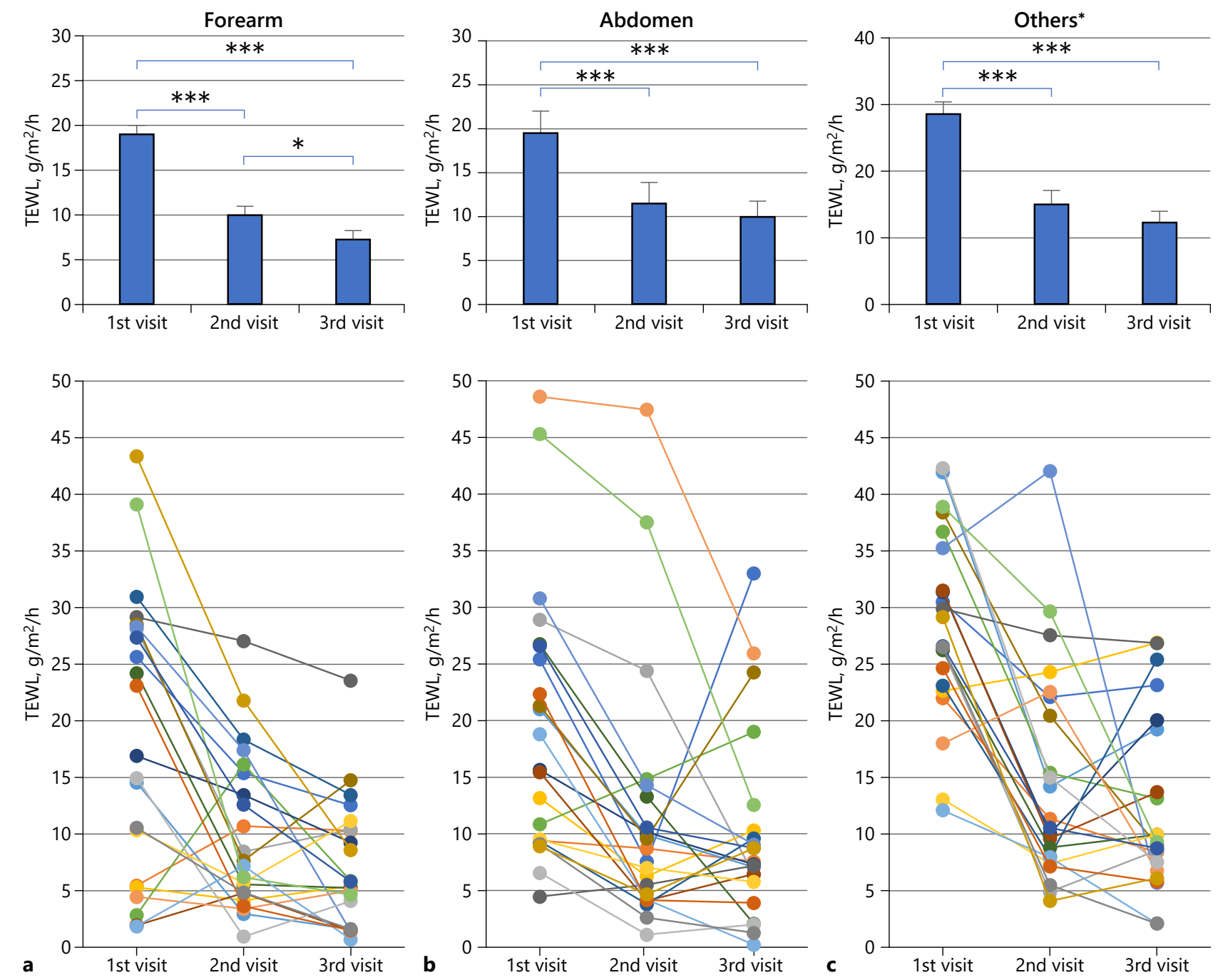

Fig. 4. Changes in TEWL before, during, and after topical treatment. TEWL was evaluated at the first, second, and third visits for the forearm, abdomen, and area with the most severe symptoms (others*). The average data are indicated by the bar graphs at the top, and individual data are indicated by the line graphs below. Data are expressed as the mean \pm SEM. ${ }^{*} p<0.05,{ }^{* * *} p<0.001$. TEWL, transepidermal water loss; SEM, standard error of the mean.

\section{Statistical Analysis}

Student's $t$ test and Mann-Whitney's U test were used to compare scIL- 8 levels between the 2 groups, and Spearman's rank correlation test was used to calculate the correlations. Results are expressed as the mean \pm standard error of the mean, unless otherwise indicated. The results were considered to be significantly different or correlated when the $p$ value $<0.05$.

\section{Results}

\section{Patient Demographics and Clinical Characteristics}

The mean \pm standard deviation age of the overall cohort was $28.5 \pm 9.9$ years. Of the 22 patients, 13 had severe symptoms (SCORAD >50), 7 had moderate symptoms (SCORAD 25-50), and 2 had mild symptoms (SCORAD $<25)$. The mean \pm standard deviation SCORAD score was $52.6 \pm 17.0$ (Table 1$)$. 


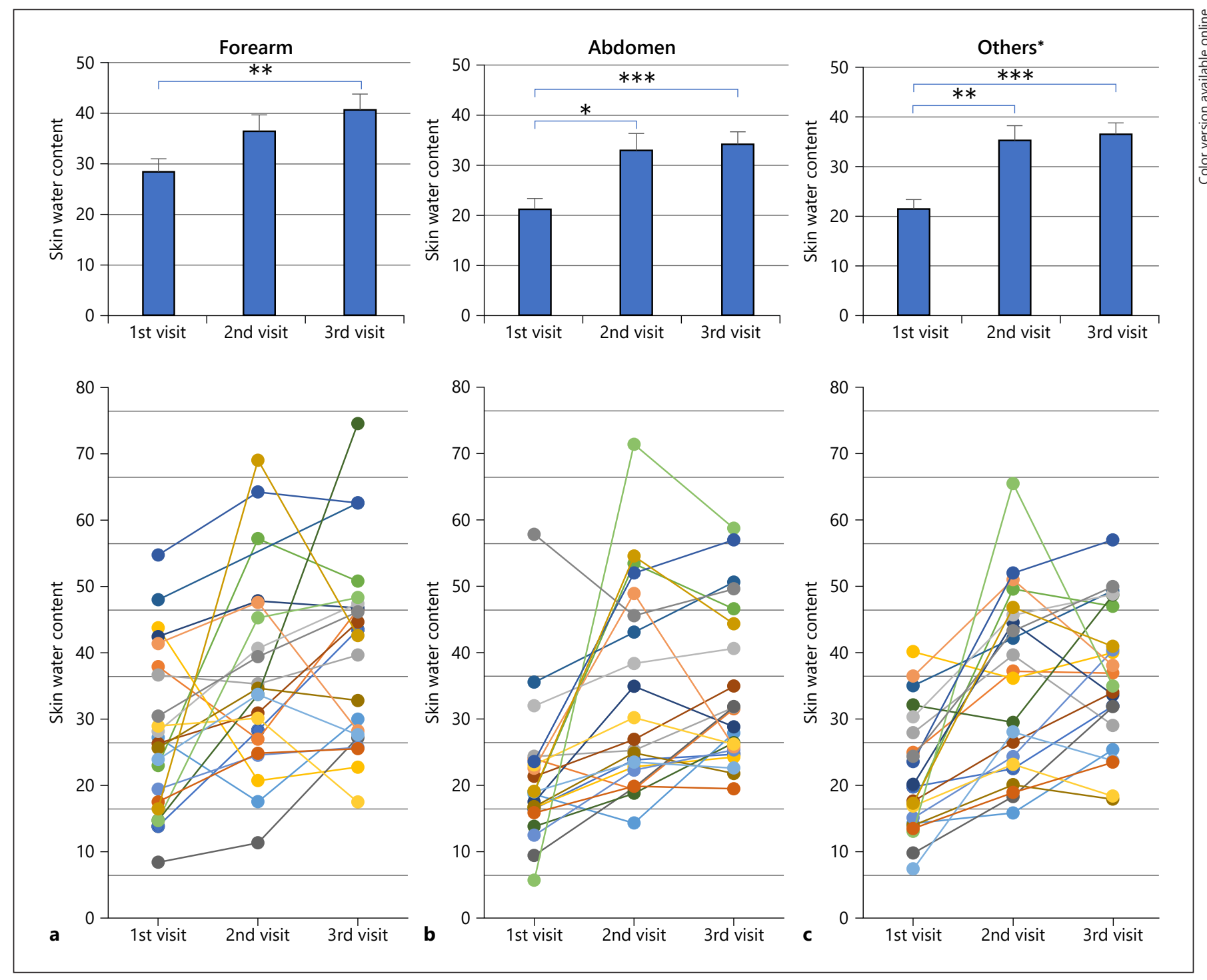

Fig. 5. Changes in skin water content before, during, and after topical treatment. Skin water content was evaluated at the first, second, and third visits for the forearm, abdomen, and area with the most severe symptoms (others*). The average data are indicated by the bar graphs at the top, and individual data are indicated by the line graphs below. Data are expressed as the mean \pm SEM. ${ }^{*} p<0.05,{ }^{* *} p<0.01,{ }^{* * *} p<0.001$. SEM, standard error of the mean.

Correlation between scIL-8 and Skin Scores, TEWL, and Skin Water Content before Topical Treatment

The average scIL- 8 concentration in the patients before the treatment was $790 \pm 348 \mathrm{pg} / \mathrm{mg}$ on the forearm, $902 \pm 391 \mathrm{pg} / \mathrm{mg}$ on the abdomen, and $1,905 \pm 500 \mathrm{pg} / \mathrm{mg}$ over the lesions with the most severe symptoms. The correlation between scIL- 8 and skin scores at the 3 sites is illustrated in Fig. 2a-c. Significant correlations were observed between scIL-8 and skin score on the forearm $\left(r_{\mathrm{s}}=0.50, p<0.001\right)$, abdomen $\left(r_{\mathrm{s}}=0.37, p<0.01\right)$, and area with the most severe symptoms $\left(r_{\mathrm{s}}=0.53, p<0.001\right)$. The correlation between scIL- 8 and TEWL in the same areas before topical treatment is illustrated in Fig. 2d-f. A significant correlation was observed between scIL- 8 and TEWL in the forearm $\left(r_{s}=0.45, p<0.05\right)$, abdomen $\left(r_{s}=\right.$ $0.69, p<0.01)$, and area with the most severe symptoms $\left(r_{\mathrm{s}}=0.42 . p<0.05\right)$. However, no statistically significant correlation was found between scIL-8 and skin water content in the same areas (Fig. 2g-i). 
Table 2. Total skin score, TEWL, skin water content, and scIL-8 concentration over the test period for the forearm, abdomen, and area with the most severe symptoms (others*)

\begin{tabular}{lrrr}
\hline & First visit & Second visit & Third visit \\
\hline scIL-8, pg/mg & & & \\
Forearm & $790.3 \pm 348.0$ & $162.9 \pm 67.9$ & $99.6 \pm 37.0$ \\
Abdomen & $902.1 \pm 391.4$ & $165.0 \pm 56.6$ & $142.0 \pm 38.4$ \\
Others* & $1,904.8 \pm 499.6$ & $267.1 \pm 108.3$ & $242.8 \pm 65.1$ \\
\hline Total skin score & & & \\
Forearm & $7.8 \pm 1.0$ & $4.0 \pm 0.7$ & $2.0 \pm 0.4$ \\
Abdomen & $7.3 \pm 0.9$ & $1.9 \pm 0.4$ & $1.5 \pm 0.3$ \\
Others* & $10.4 \pm 0.9$ & $3.5 \pm 0.6$ & $2.5 \pm 0.5$ \\
\hline TEWL, g/m ${ }^{2} / h$ & & & \\
Forearm & $19.0 \pm 2.7$ & $9.9 \pm 1.5$ & $7.2 \pm 1.2$ \\
Abdomen & $19.5 \pm 2.5$ & $11.4 \pm 2.4$ & $9.9 \pm 1.8$ \\
Others* & $28.5 \pm 1.8$ & $14.9 \pm 2.1$ & $12.2 \pm 1.7$ \\
\hline Skin water content & & & \\
Forearm & $28.3 \pm 2.6$ & $36.4 \pm 3.2$ & $40.6 \pm 3.1$ \\
Abdomen & $21.1 \pm 2.2$ & $32.9 \pm 3.4$ & $34.1 \pm 2.5$ \\
Others* & $21.4 \pm 1.9$ & $35.2 \pm 3.0$ & $36.4 \pm 2.3$ \\
\hline
\end{tabular}

Data are expressed as the mean \pm standard error of the mean. TEWL, transepidermal water loss; scIL-8, stratum corneum interleukin-8.

Skin Score, TEWL, and Skin Water Content before, during, and after Topical Treatment

All 22 patients completed this study. The average skin score, TEWL, and skin water content before, during, and after topical treatment are shown in Figures 3-5. Skin scores decreased significantly at the second and third visits compared to those at the first visit at all 3 sites (Fig. 3). Additionally, the average TEWL values decreased significantly at the second and third visits compared to those at the first visit at all 3 sites (Fig. 4). Skin water content increased significantly at the third visit compared to those at the first visit at all 3 sites (Fig. 5). The actual average skin score, TEWS, and skin water content throughout the test period are summarized in Table 2 .

\section{Changes in Laboratory Parameters before and after Topical Treatment}

The mean serum levels of TARC, total IgE, LDH, and \%eosinophil before and after the topical treatment are summarized in Figure 6. The serum levels of TARC and LDH decreased significantly at the third visit, whereas that of serum total IgE did not change significantly. The blood \%eosinophil decreased significantly at the third visit.

Interleukin-8 in Stratum Corneum as a Biomarker of Atopic Dermatitis
Changes in scIL-8 before and after Topical Treatment

The average levels of scIL- 8 before, during, and after topical treatment are presented in Figure 7 and Table 2. scIL-8 levels on the forearm, abdomen, and on the skin lesion with the most severe symptoms decreased significantly from the first visit to the second and third visits. The highest reduction in scIL-8 levels was between the first and third visits on the skin lesion with the most severe symptom.

\section{Correlation between scIL-8 Reduction and Skin Score} Improvement with Topical Treatment

The correlation between the reduction in scIL-8 levels $(\Delta$ scIL-8) and the degree of improvements in the skin score ( $\Delta$ skin score) following the topical treatment is shown in Figure 8. $\Delta$ scIL-8 (difference between the values at first and third visits) was significantly correlated with the $\Delta$ skin score (difference between the values at first and third visits $)$ in the forearm $\left(r_{\mathrm{s}}=0.50, p<0.01\right)$, abdomen $\left(r_{\mathrm{s}}=0.82, p<0.001\right)$, and area with the most severe symptoms $\left(r_{\mathrm{s}}=0.55, p<0.01\right)$. Similar significant correlations were observed between the $\Delta$ scIL-8 (difference between the values at first and second visits) and the $\Delta$ skin score (difference between the values at first and second visits) for all 3 sites, and between the $\Delta$ scIL- 8 (difference in the values at second and third visits) and $\Delta$ skin score (difference in the values at second and third visits) for the abdomen and area with the most severe symptoms (Fig. 8).

\section{Correlation between scIL-8 Reduction and}

Improvements in TEWL and Skin Water Content

following Topical Treatment

The correlation between the $\Delta$ scIL- 8 and the degrees of improvement in TEWL ( $\triangle \mathrm{TEWL})$ and skin water content ( $\Delta$ skin water content) following topical treatment is illustrated in Figure 9. When the $\Delta$ scIL-8 and $\triangle \mathrm{TEWL}$ were analyzed between the first and third visits, there were no significant correlations in the forearm $\left(r_{\mathrm{s}}=0.16\right)$, abdomen $\left(r_{\mathrm{s}}=0.33\right)$, or area with the most severe symptoms $\left(r_{s}=0.21\right)$. However, when $\Delta$ scIL- 8 and $\Delta$ skin water content were analyzed between the first and third visits, a significant correlation was observed in the abdomen $\left(r_{\mathrm{s}}=\right.$ $0.41, p<0.05)$.

\section{Correlation between Reduction in scIL-8 and}

Improvements in the General Severity Parameters

following Topical Treatment

The correlation between $\Delta$ scIL- 8 and the improvements in serum levels of TARC ( $\triangle$ TARC), \%eosinophil $(\Delta \%$ eosinophil), and $\mathrm{LDH}(\Delta \mathrm{LDH})$ following topical treat- 


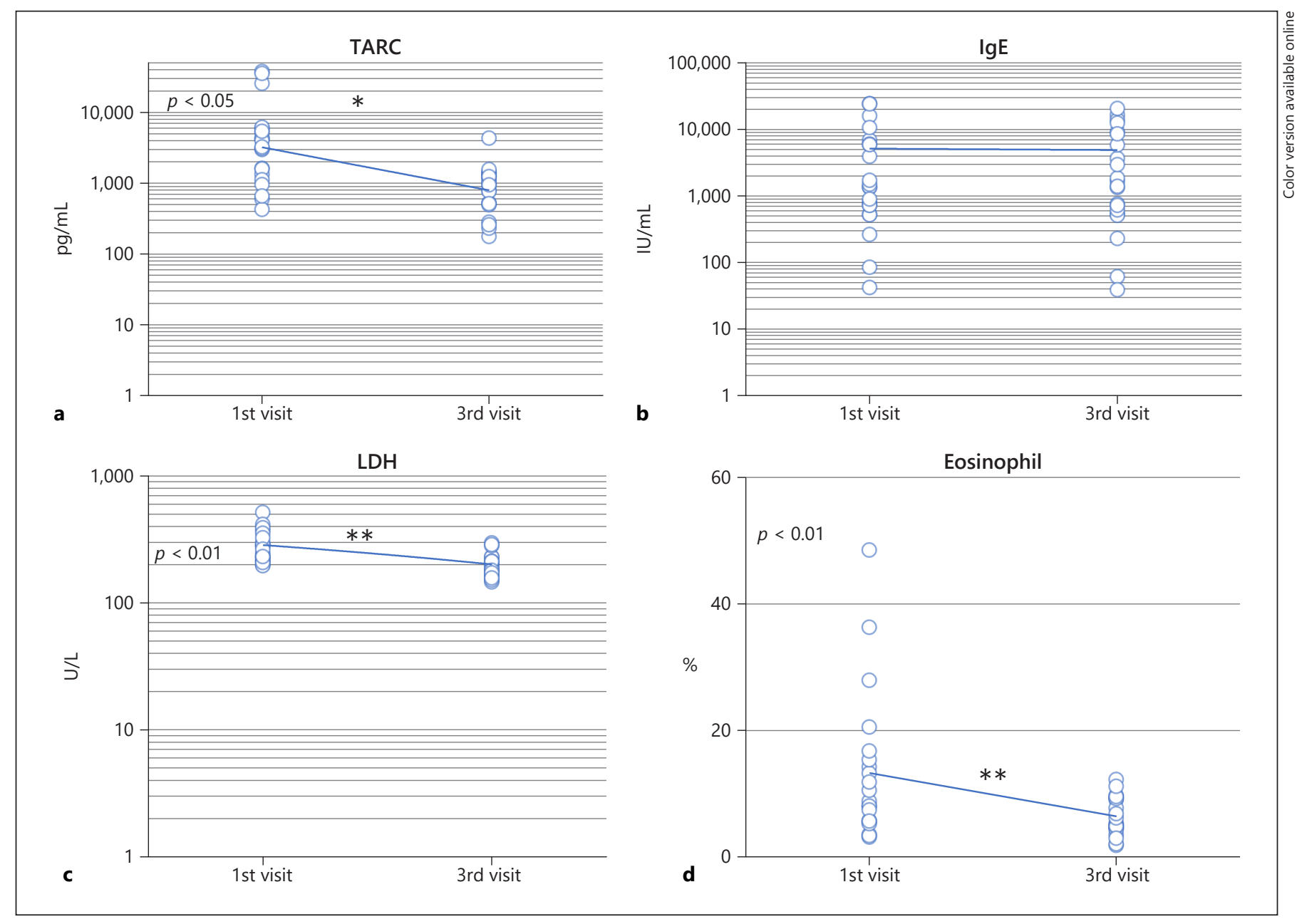

Fig. 6. Laboratory data before and after treatment. Levels of serum TARC, serum total IgE, serum LDH, and blood \%eosinophil were evaluated at the first and third visits. ${ }^{*} p<0.05,{ }^{* *} p<0.01$. TARC, thymus and activation-regulated chemokine; IgE, immunoglobulin E; LDH, lactate dehydrogenase.

ment is presented in Figure 10. Significant correlations were noted between $\Delta$ scIL- 8 and $\triangle$ TARC in the forearm $\left(r_{\mathrm{s}}=0.65, p<0.01\right)$ and abdomen $\left(r_{\mathrm{s}}=0.53, p<0.01\right)$, between $\Delta$ scIL-8 and $\Delta \%$ eosinophil in the abdomen $\left(r_{s}=0.50\right.$, $p<0.05)$, and between $\Delta$ scIL-8 and $\Delta \mathrm{LDH}$ in the forearm $\left(r_{\mathrm{s}}=0.39, p<0.05\right)$ and abdomen $\left(r_{\mathrm{s}}=0.54, p<0.01\right)$. No significant correlations were noted between $\Delta$ scIL-8 and improvement in serum IgE levels (data not shown).

\section{Discussion/Conclusion}

This study demonstrated that scIL-8, measured using the tape-stripping method, reflected the response to topical corticosteroid therapy in $\mathrm{AD}$ patients; further, the de- gree of change in scIL-8 concentration was correlated with visual improvements in symptoms.

Before the topical corticosteroid treatment, the scIL-8 concentration at lesion sites correlated with the visual skin score, which is consistent with the previous observations by McAleer et al. [15] and Hulshof et al. [16], as well as with our previous results [11]. McAleer et al. [15] reported that 19 cytokines, including IL-8, demonstrated significant differences between healthy subjects and infants with $\mathrm{AD}$; additionally, they showed that the levels of IL-8 and IL-18 were the highest among cytokines measured in the stratum corneum. Hulshof et al. [16] demonstrated that IL-8, CCL2, and TARC measured using the tape-stripping method in children with $\mathrm{AD}$ showed an association in the objective SCORAD score. These cumu- 


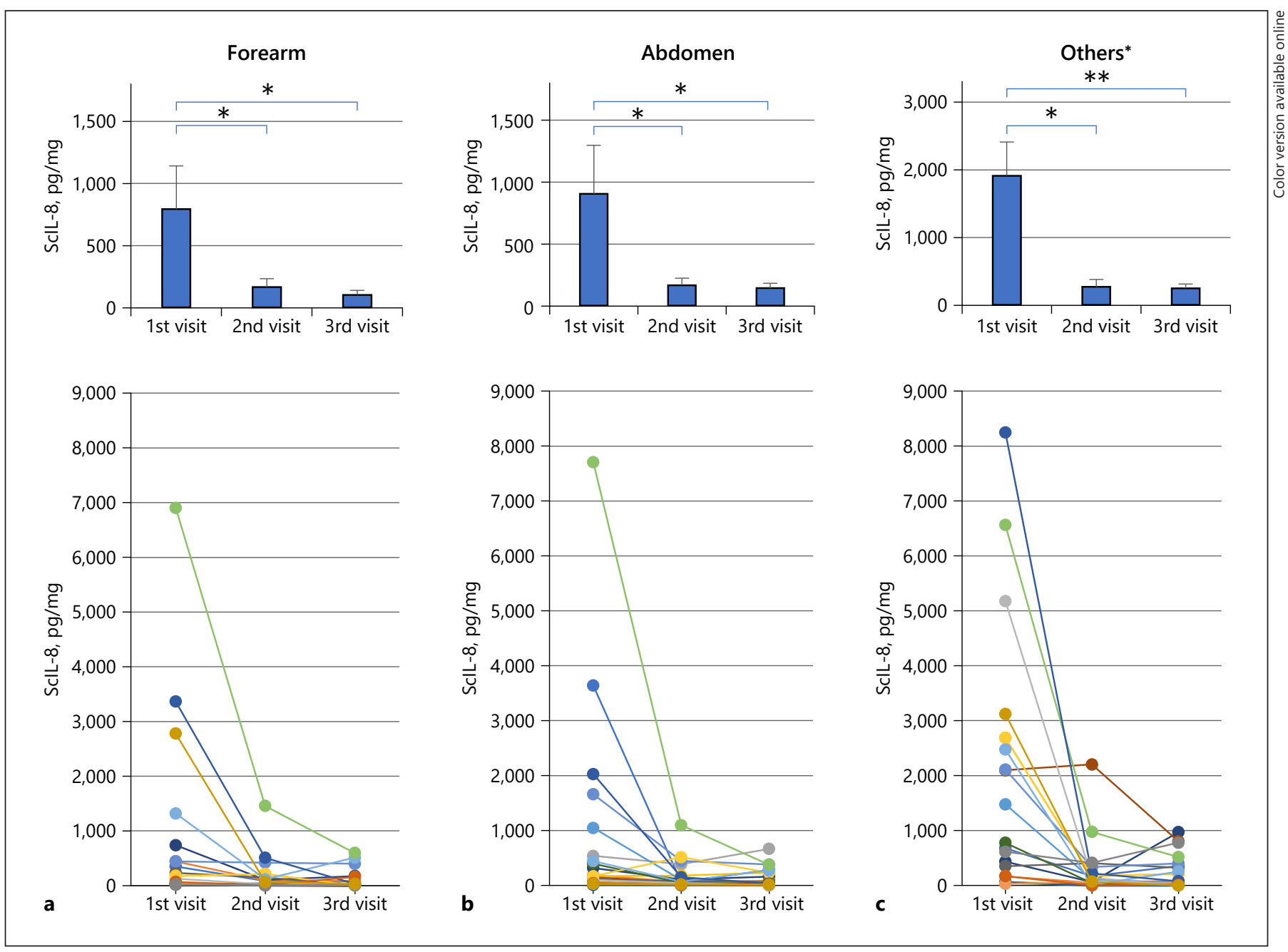

Fig. 7. Changes in scIL-8 level before, during, and after topical treatment. scIL- 8 was evaluated at the first, second, and third visits on the forearm, abdomen, and area with the most severe symptoms (others*). The average data are indicated in the bar graphs at the top, and individual data are indicated in the line graphs below. Significant reductions in scIL-8 levels were observed following topical corticosteroid treatment at all 3 skin sites. Data are expressed as the mean \pm standard error of the mean. ${ }^{*} p<0.05,{ }^{* *} p<0.01$. scIL-8, stratum corneum interleukin-8.

lative findings suggest that assessment of scIL- 8 is a useful tool in evaluating the severity of skin inflammation in $\mathrm{AD}$ patients; however, data on the change in scIL-8 level with pharmaceutical intervention are lacking. Topical corticosteroid treatment is the preferred first-line therapy for $\mathrm{AD}$, as recommended in the guidelines by the Japanese, American, and European Academies of Dermatology [12-14]. Koppes et al. [17] investigated the effects of 6 weeks of ceramide- and magnesium-containing emollient therapy on 38 inflammatory mediators in the stratum corneum in mild and moderate $\mathrm{AD}$ patients. They reported that decreases in TARC and IL- 8 were corre- lated with the decrease of disease severity in the subgroup of moderate $\mathrm{AD}$ individuals. In their study, patients with severe $\mathrm{AD}$ were excluded, and patients were not allowed to apply topical corticosteroids. In the present study, we demonstrated that changes in scIL-8 levels reflect pharmacologic responses to topical corticosteroids for improvement of clinical AD symptoms. To the best of our knowledge, this study is the first to demonstrate the usefulness of scIL- 8 determination in evaluating improvements of skin lesions in patients with $\mathrm{AD}$ through daily topical corticosteroid treatment. Following topical corticosteroid treatment, skin score improved significantly, as 


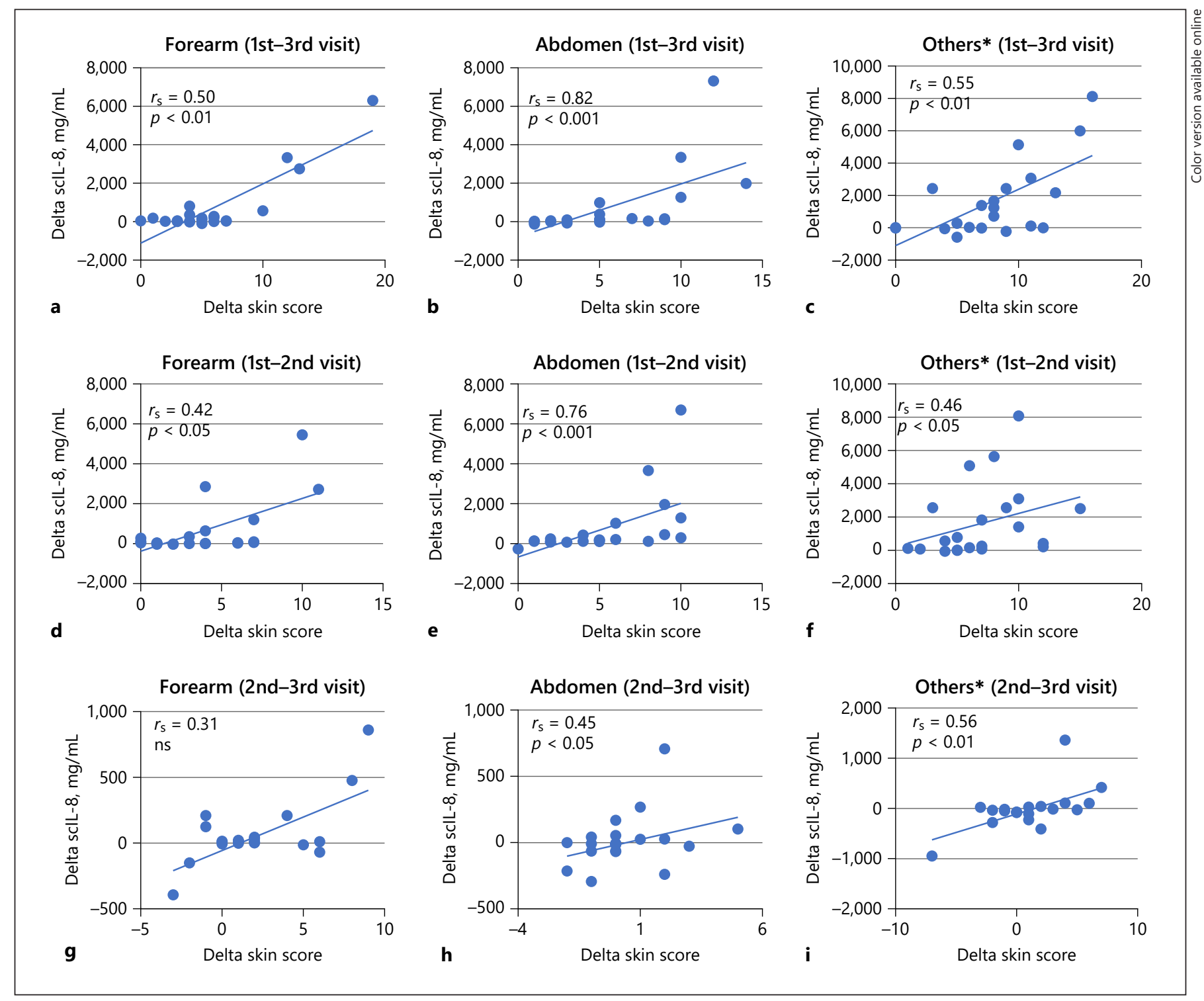

Fig. 8. Correlation between delta scIL- 8 and delta skin score at the forearm, abdomen, and area with the most severe symptoms (others*). The values were calculated at the first to third visits, first to second visits, and second to third visits. scIL-8, stratum corneum interleukin-8; NS, not significant.

indicated in Figures 3, and the scIL-8 level decreased significantly, as indicated in Figure 7. In addition, the degree of skin symptom improvement ( $\Delta$ skin score) was correlated with $\Delta$ scIL-8 (Fig. 8). It is noteworthy that the higher correlation coefficients were observed between $\Delta$ scIL-8 and the $\Delta$ skin score upon subgroup analysis of patients with severe $\mathrm{AD}$ (SCORAD $>50, n=14$ ) between the first and third visits. The $r_{\mathrm{s}}$ values were as follows: forearm, $0.63(p<0.01)$; abdomen, $0.80(p<0.01)$; and area with the most severe symptoms, $0.73(p<0.01)$ (data not shown). This stronger correlation in the subgroup with severe $\mathrm{AD}$ is consistent with the study done by Koppes et al. [17] with topical emollient treatment. In our previous study, we described that scIL-8 correlates highly with acute phase symptoms, such as erythema, edema/papules, and excoriation; however, it is weaker with chronic phase symptoms, such as lichenification and oozing/crust [11]. This could be one of the potential reasons why correlation between scIL-8 level and visual skin score is low in mild $\mathrm{AD}$ where chronic phase symptoms are predom- 


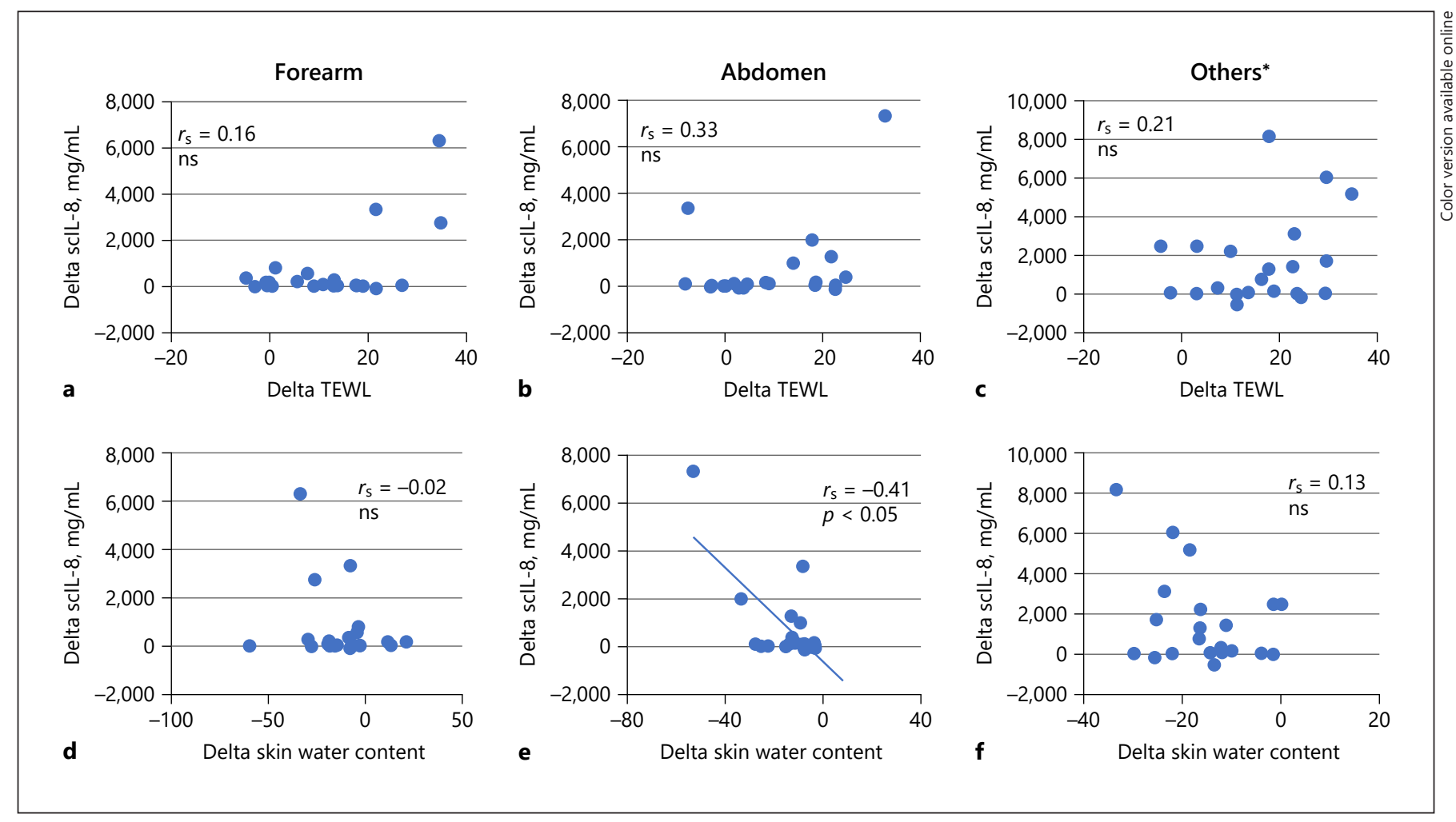

Fig. 9. Correlation between delta scIL-8 and delta TEWL and delta skin water content, respectively, at the forearm, abdomen, and the area with the most severe symptoms (others*) for the first to third visits. NS, not significant. scIL-8, stratum corneum interleukin-8; TEWL, transepidermal water loss.

inant and correlation between scIL-8 level and visual skin score is high in severe $\mathrm{AD}$ patients where acute phase symptoms are predominant.

As we have previously reported, scIL-8 levels are extremely low in persons without $\mathrm{AD}$ - almost under the detection limit of the commercially available ELISA kit; in comparison, such levels are increased up to 100 times and more in patients with $\mathrm{AD}[10,11]$. This is in agreement with the present results, in which all patients with AD demonstrated detectable levels of scIL- 8 on the forearm, abdomen, and skin affected worst with symptoms. Paralleling improvement in skin symptoms, scIL-8 levels drastically decreased after 2 weeks of topical treatment and remained low until at least 4-6 weeks of treatment (Fig. 5). It should be noted that scIL-8 levels were still detectable after 4-6 weeks of topical treatment in most patients. Only $2 / 22,2 / 22$, and $2 / 22$ patients did not demonstrate detectable levels of scIL-8, respectively, on the forearm, abdomen, and the lesion sites with the most severe symptoms. For these sites, there were $7 / 22,6 / 22$, and $5 / 22$ patients, respectively, with a skin score of 0 . These results suggest that scIL- 8 has high sensitivity to reflect improvements in local inflammation in patients with $\mathrm{AD}$, more so than visual skin scoring.

We discovered that scIL-8 was weakly correlated with TEWL, not with skin water content (Fig. 2), although our previous findings demonstrated that scIL-8 was associated with both TEWL and skin water content. This might be due to the number of patients investigated: 22 in this study compared to 55 in the previous study [11]. $\Delta$ scIL-8 did not show a correlation with $\triangle \mathrm{TEWL}$ or $\Delta$ skin water content in this study, thus suggesting that scIL-8 may not be a sensitive biomarker in evaluating the improvements in barrier damage due to $\mathrm{AD}$.

Additionally, scIL-8 might reflect systemic disease severity of $\mathrm{AD}$, especially when it is evaluated on the forearm or abdomen, since $\Delta$ scIL- 8 was correlated with serum $\triangle \mathrm{TARC}$ and $\triangle \mathrm{LDH}$ levels, which are established biomarkers of severity in AD (Fig. 10). During topical treatment, no significant change was observed in total serum IgE levels although serum levels of TARC, LDH, and \%eosinophil declined significantly (Fig. 6). This is consis- 


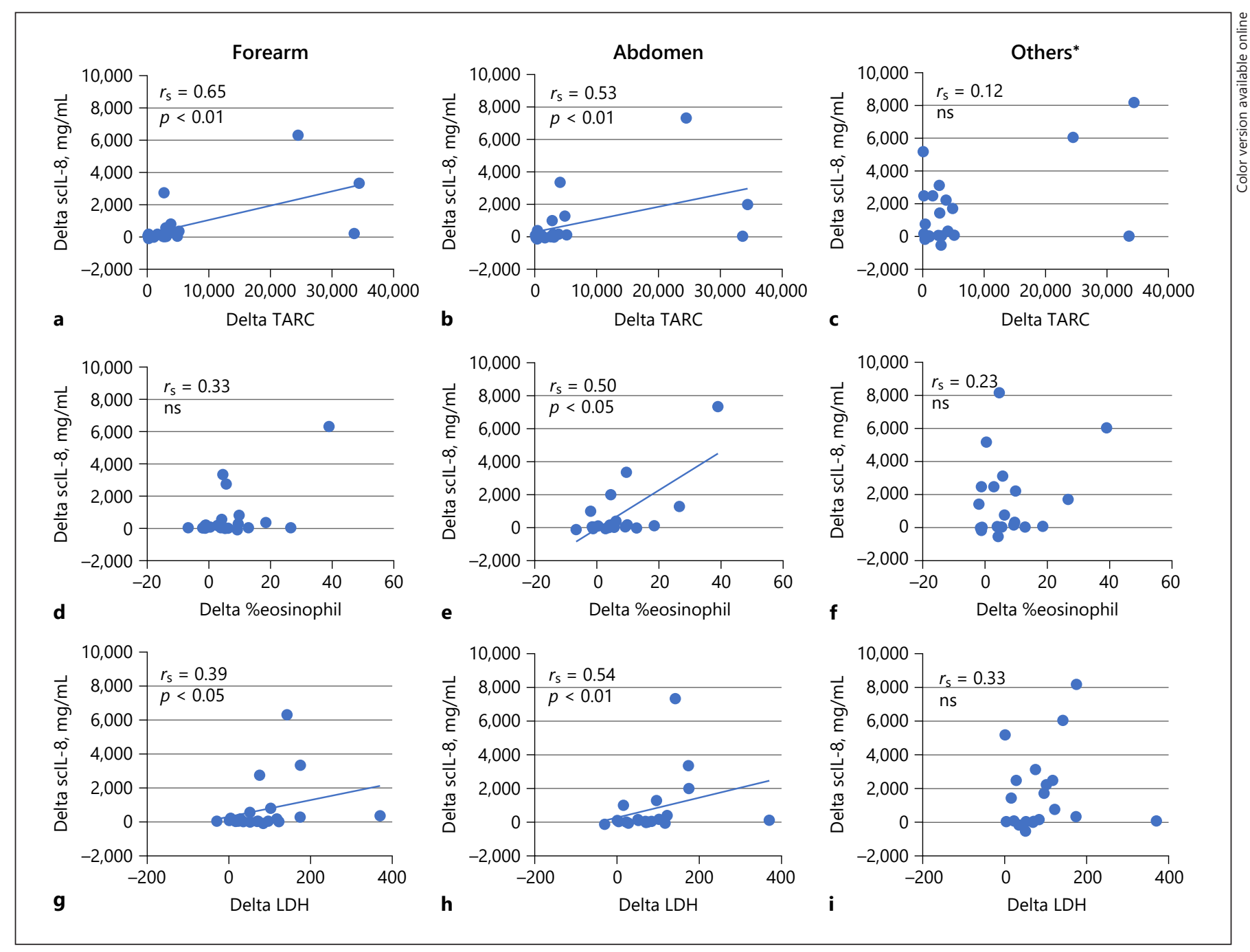

Fig. 10. Comparison between delta scIL- 8 and delta serum levels of TARC, \%eosinophil, and LDH at the forearm, abdomen, and area with the most severe symptoms (others*) for the first to third visits. Significant correlations were observed between delta scIL- 8 and delta TARC, delta \%eosinophil, and delta LDH. scIL-8, stratum corneum interleukin-8; TARC, thymus and activation-regulated chemokine; LDH, lactate dehydrogenase; NS, not significant.

tent with the findings of previous studies, in which it was reported that total serum IgE levels correlated with the severity of $\mathrm{AD}$ but did not decrease proportionally with improvements in AD [18-20]. Although scIL-8 level correlated significantly above serum biomarkers, these correlation coefficients were relatively low. It may be a reasonable assumption that scIL-8 serves as biomarker for local skin severity of $\mathrm{AD}$ more than systemic inflammation, whereas serum blood markers reflect more systemic inflammation in $\mathrm{AD}$ patients.
The tape-stripping technique has been established as a noninvasive and relatively quick and simple method for estimating cytokine concentrations in the stratum corneum [21-25]. In this study, we successfully obtained the stratum corneum from the lesions before, during, and after topical treatment.

IL-8 is a pro-inflammatory chemokine and a potent chemoattractant for neutrophils, playing a role in the activation of the innate immunity. IL- 8 was identified originally as a neutrophil-activating peptide or human monocyte-derived neutrophil chemotactic factor from super- 
natants obtained from activated monocytes [26-29]. This monokine was demonstrated to have chemotactic activity for T lymphocytes and was later renamed as IL-8 [30, 31]. The production of large amounts of IL- 8 in psoriasis has also been demonstrated repeatedly by Schröder et al. [32-35]. Thus, scIL-8 is not specific to AD and could be used to monitor other diseases related to skin inflammation. Therefore, the measurement of scIL- 8 using the tape-stripping method may also be applied in evaluating improvements in the severity of lesions in psoriasis or other inflammation-related skin diseases.

The limitations of this study are its relatively small sample size $(n=22)$ and the absence of potent pharmaceutical treatments other than topical corticosteroid treatment. While topical corticosteroid therapy has been the standard therapy for $\mathrm{AD}$, other treatments including topical calcineurin inhibitors, systemic oral cyclosporine, and dupilumab injection therapy can be used to treat $\mathrm{AD}$. The present study did not demonstrate a change in scIL8 levels secondary to these therapies or other emerging treatments, including those that mitigate the JAK-STAT pathway and PDE4 enzyme inhibition [36, 37].

The degree of scIL-8 change, estimated using the noninvasive tape-stripping method, reflects improvement in skin symptoms following first-line $\mathrm{AD}$ treatment with topical corticosteroids. Thus, it may be a valuable biomarker to monitor therapeutic effect in $\mathrm{AD}$ patients.

\section{Acknowledgements}

We thank all the participating medical practices for the recruitment of patients and for their support of this clinical study.

\section{Statement of Ethics}

This study was approved by the Ethics Committee of Shimane University Faculty of Medicine (Approval No. 1473) and was performed in accordance with the Declaration of Helsinki. The study design was fully explained to the patients, and written informed consent was obtained from them.

\section{Conflict of Interest Statement}

The authors have no conflicts of interest to declare.

\section{Funding Sources}

The authors did not receive any funding.

\section{Author Contributions}

All authors contributed to editing and reviewing of the draft manuscript and provided approval of the final version of the manuscript.

\section{References}

1 Tsai TF, Rajagopalan M, Chu CY, Encarnacion L, Gerber RA, Santos-Estrella P, et al. Burden of atopic dermatitis in Asia. J Dermatol. 2019 Oct; 46(10):825-34.

2 Silvestre Salvador JF, Romero-Pérez D, Encabo-Durán B. Atopic dermatitis in adults: a diagnostic challenge. J Investig Allergol Clin Immunol. 2017;27(2):78-88

3 Tamaki K, Kakinuma T, Saeki H, Horikawa T, Kataoka Y, Fujisawa T, et al. Serum levels of CCL17/TARC in various skin diseases. J Dermatol. 2006 Apr;33(4):300-2.

4 Jahnz-Rozyk K, Targowski T, Paluchowska E, Owczarek W, Kucharczyk A. Serum thymus and activation-regulated chemokine, macrophage-derived chemokine and eotaxin as markers of severity of atopic dermatitis. Allergy. 2005 May;60(5):685-8.

5 Thijs J, Krastev T, Weidinger S, Buckens CF, de Bruin-Weller M, Bruijnzeel-Koomen C, et al. Biomarkers for atopic dermatitis: a systematic review and meta-analysis. Curr Opin $\mathrm{Al}-$ lergy Clin Immunol. 2015 Oct;15(5):453-60.

Interleukin-8 in Stratum Corneum as a Biomarker of Atopic Dermatitis
6 Morishima Y, Kawashima H, Takekuma K, Hoshika A. Changes in serum lactate dehydrogenase activity in children with atopic dermatitis. Pediatr Int. 2010 Apr;52(2):171-4.

7 Perkins MA, Osterhues MA, Farage MA, Robinson MK. A noninvasive method to assess skin irritation and compromised skin conditions using simple tape adsorption of molecular markers of inflammation. Skin Res Technol. 2001 Nov;7(4):227-37.

8 Morita E, Hiragun T, Mihara S, Kaneko S, Matsuo H, Zhang Y, et al. Determination of thymus and activation-regulated chemokine (TARC)-contents in scales of atopic dermatitis. J Dermatol Sci. 2004 May;34(3):237-40.

9 Morita E, Takahashi H, Niihara H, Dekio I, Sumikawa Y, Murakami Y, et al. Stratum corneum TARC level is a new indicator of lesional skin inflammation in atopic dermatitis. Allergy. 2010 Sep;65(9):1166-72.

10 Amarbayasgalan T, Takahashi H, Dekio I, Morita E. Content of vascular endothelial growth factor in stratum corneum well correlates to local severity of acute inflammation in patients with atopic dermatitis. Int Arch Allergy Immunol. 2012;157(3):251-8.
11 Amarbayasgalan T, Takahashi H, Dekio I, Morita E. Interleukin-8 content in the stratum corneum as an indicator of the severity of inflammation in the lesions of atopic dermatitis. Int Arch Allergy Immunol. 2013;160(1): 63-74.

12 Wollenberg A, Barbarot S, Bieber T, ChristenZaech S, Deleuran M, Fink-Wagner A, et al. Consensus-based European guidelines for treatment of atopic eczema (atopic dermatitis) in adults and children: part I. J Eur Acad Dermatol Venereol. 2018 May;32(5):657-82.

13 Eichenfield LF, Tom WL, Berger TG, Krol A, Paller AS, Schwarzenberger K, et al. Guidelines of care for the management of atopic dermatitis: section 2. Management and treatment of atopic dermatitis with topical therapies. J Am Acad Dermatol. 2014 Jul;71(1): 116-32.

14 Aoki T, Yoshida H, Furue M, Tagami H, Kaneko F, Ohtsuka F, et al. English version of the concluding report published in 2001 by the Advisory Committee on Atopic Dermatitis Severity Classification Criteria of the Japanese Dermatological Association. J Dermatol. 2011 Jul;38(7):632-44. 
15 McAleer MA, Jakasa I, Hurault G, Sarvari P, McLean WHI, Tanaka RJ, et al. Systemic and stratum corneum biomarkers of severity in infant atopic dermatitis include markers of innate and $\mathrm{T}$ helper cell-related immunity and angiogenesis. Br J Dermatol. 2019 Mar; 180(3):586-96.

16 Hulshof L, Hack DP, Hasnoe QCJ, Dontje B, Jakasa I, Riethmüller C, et al. A minimally invasive tool to study immune response and skin barrier in children with atopic dermatitis. Br J Dermatol. 2019 Mar;180(3):621-30.

17 Koppes SA, Brans R, Ljubojevic Hadzavdic S, Frings-Dresen $\mathrm{MH}$, Rustemeyer T, Kezic S. Stratum corneum tape stripping: monitoring of inflammatory mediators in atopic dermatitis patients using topical therapy. Int $\mathrm{Arch} \mathrm{Al}-$ lergy Immunol. 2016;170(3):187-93.

18 Stevens SR, Hanifin JM, Hamilton T, Tofte SJ, Cooper KD. Long-term effectiveness and safety of recombinant human interferon gamma therapy for atopic dermatitis despite unchanged serum IgE levels. Arch Dermatol. 1998 Jul;134(7):799-804.

19 Kou K, Aihara M, Matsunaga T, Chen H, Taguri M, Morita S, et al. Association of serum interleukin-18 and other biomarkers with disease severity in adults with atopic dermatitis. Arch Dermatol Res. 2012 May;304(4): 305-12.

20 Thijs JL, van Seggelen W, Bruijnzeel-Koomen C, de Bruin-Weller M, Hijnen D. New developments in biomarkers for atopic dermatitis. J Clin Med. 2015 Mar 16;4(3):479-87.

21 Akdis CA, Akdis M. Immunological differences between intrinsic and extrinsic types of atopic dermatitis. Clin Exp Allergy. 2003 Dec; 33(12):1618-21.

22 Guttman-Yassky E, Diaz A, Pavel AB, Fernandes M, Lefferdink R, Erickson T, et al. Use of tape strips to detect immune and barrier abnormalities in the skin of children with early-onset atopic dermatitis. JAMA Dermatol. 2019 Oct 9;155(12):1358-70.
23 Kezic S, Kammeyer A, Calkoen F, Fluhr JW, Bos JD. Natural moisturizing factor components in the stratum corneum as biomarkers of filaggrin genotype: evaluation of minimally invasive methods. Br J Dermatol. 2009 Nov; 161(5):1098-104.

24 Breternitz M, Flach M, Prässler J, Elsner P, Fluhr JW. Acute barrier disruption by adhesive tapes is influenced by pressure, time and anatomical location: integrity and cohesion assessed by sequential tape stripping. A randomized, controlled study. Br J Dermatol. 2007 Feb;156(2):231-40.

25 de Jongh CM, Verberk MM, Spiekstra SW, Gibbs S, Kezic S. Cytokines at different stratum corneum levels in normal and sodium lauryl sulphate-irritated skin. Skin Res Technol. 2007 Nov; 13(4):390-8.

26 Schröder JM, Mrowietz U, Morita E, Christophers E. Purification and partial biochemical characterization of a human monocyte-derived, neutrophil-activating peptide that lacks interleukin 1 activity. J Immunol. 1987 Nov 15;139(10):3474-83.

27 Matsushima K, Morishita K, Yoshimura T, Lavu S, Kobayashi Y, Lew W, et al. Molecular cloning of a human monocyte-derived neutrophil chemotactic factor (MDNCF) and the induction of MDNCF mRNA by interleukin 1 and tumor necrosis factor. J Exp Med. 1988 Jun 1;167(6):1883-93.

28 Van Damme J, Van Beeumen J, Opdenakker G, Billiau A. A novel, NH2-terminal sequence-characterized human monokine possessing neutrophil chemotactic, skin-reactive, and granulocytosis-promoting activity. J Exp Med. 1988 Apr 1;167(4):1364-76.
29 Peveri P, Walz A, Dewald B, Baggiolini M. A novel neutrophil-activating factor produced by human mononuclear phagocytes. J Exp Med. 1988 May 1;167(5):1547-59.

30 Larsen CG, Anderson AO, Appella E, Oppenheim JJ, Matsushima K. The neutrophil-activating protein (NAP-1) is also chemotactic for T lymphocytes. Science. 1989 Mar 17; 243(4897): 1464-6.

31 Leonard EJ, Yoshimura T. Neutrophil attractant/activation protein-1 (NAP-1 [interleukin-8]). Am J Respir Cell Mol Biol. 1990 Jun; 2(6):479-86.

32 Sticherling M, Bornscheuer E, Schröder JM, Christophers E. Immunohistochemical studies on NAP-1/IL-8 in contact eczema and atopic dermatitis. Arch Dermatol Res. 992; 284(2):82-5

33 Schröder JM, Christophers E. Identification of C5ades arg and an anionic neutrophil-activating peptide (ANAP) in psoriatic scales. J Invest Dermatol. 1986 Jul;87(1):53-8.

34 Sticherling M, Bornscheuer E, Schröder JM Christophers E. Localization of neutrophilactivating peptide-1/interleukin-8-immunoreactivity in normal and psoriatic skin. J Invest Dermatol. 1991 Jan;96(1):26-30.

35 Schröder JM, Gregory H, Young J, Christophers E. Neutrophil-activating proteins in psoriasis. J Invest Dermatol. 1992 Feb;98(2): 241-7.

36 Nygaard U, Vestergaard C, Deleuran M. Emerging treatment options in atopic dermatitis: systemic therapies. Dermatology. 2017; 233(5):344-57.

37 Broeders JA, Ahmed Ali U, Fischer G. Systematic review and meta-analysis of randomized clinical trials (RCTs) comparing topical calcineurin inhibitors with topical corticosteroids for atopic dermatitis: a 15-year experience. J Am Acad Dermatol. 2016 Aug;75(2): 410-9.e3. 